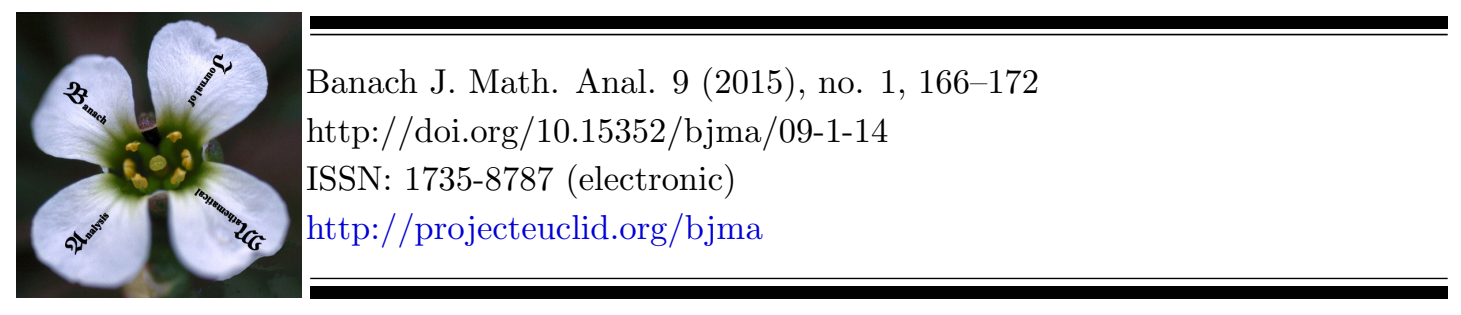

\title{
MORE OPERATOR INEQUALITIES FOR POSITIVE LINEAR MAPS
}

\author{
PINGPING ZHANG
}

Communicated by F. Kittaneh

\begin{abstract}
Some operator inequalities for positive linear maps are presented. These inequalities improve and generalize the corresponding results due to $\mathrm{Fu}$ and He [Linear Multilinear Algebra, doi: 10.1080/03081087.2014.880432.].
\end{abstract}

\section{INTRODUCTION}

As customary, we reserve $M, m$ for scalars and $I$ for the identity operator. Other capital letters denote general elements of the $C^{*}$-algebra $\mathcal{B}(\mathcal{H})$ (with unit) of all bounded linear operator acting on a Hilbert space $(\mathcal{H},\langle\cdot, \cdot\rangle)$. Also, we identify a scalar with unit multiplied by this scalar. The operator norm is denoted by $\|\cdot\|$. In this article, the inequality between operators is in the sense of Loewner partial order, that is, $T \geq S$ (the same as $S \leq T$ ) means that $T-S$ is positive. A positive invertible operator $T$ is naturally denoted by $T>0$.

A linear map $\Phi$ is positive if $\Phi(A) \geq 0$ whenever $A \geq 0$. It is said to be unital if $\Phi(I)=I$. We say that $\Phi$ is 2-positive if whenever the $2 \times 2$ operator matrix $\left[\begin{array}{cc}A & B \\ B^{*} & C\end{array}\right]$ is positive, then so is $\left[\begin{array}{cc}\Phi(A) & \Phi(B) \\ \Phi\left(B^{*}\right) & \Phi(C)\end{array}\right]$. More information on such maps can be found in [12].

The Kantorovich inequality was introduced by Kantorovich [7] (see also [6, p.444]). In 1990, the operator Kantorovich inequality was firstly established by Marshall and Olkin [10]. For a recent survey on this topic, we refer to [11]. In

Date: Received: Apr. 11, 2014; Accepted: Apr. 21, 2014.

2010 Mathematics Subject Classification. Primary 47A63; Secondary 46LXX, 47A30.

Key words and phrases. Operator inequality, positive linear map, Wielandt inequality, Kantorovich inequality, reverse AM-GM inequality. 
2013, Lin [8, Theorem 2.8] found that the operator Kantorovich inequality can be squared. This result was further generalized by $\mathrm{Fu}$ and $\mathrm{He}$, who obtained

Theorem 1.1. [5, Theorem 3] Let $0<m \leq A \leq M$. Then for every positive unital linear map $\Phi$,

$$
\Phi\left(A^{-1}\right)^{p} \leq\left(\frac{(M+m)^{2}}{4^{\frac{2}{p}} M m}\right)^{p} \Phi(A)^{-p}, \quad p \geq 2 .
$$

It is interesting to ask whether the inequality (1.1) can be improved. This is a main motivation for the present paper.

Let $A$ and $B$ be positive operators with $0<m \leq A, B \leq M$ and $\Phi$ be a positive unital linear map. The geometric mean of $A$ and $B$ is defined by

$$
A \sharp B=A^{\frac{1}{2}}\left(A^{-\frac{1}{2}} B A^{-\frac{1}{2}}\right)^{\frac{1}{2}} A^{\frac{1}{2}} .
$$

Lin [9, Theorem 2.1] obtained:

$$
\Phi\left(\frac{A+B}{2}\right)^{2} \leq\left(\frac{(M+m)^{2}}{4 M m}\right)^{2} \Phi(A \sharp B)^{2},
$$

and

$$
\Phi\left(\frac{A+B}{2}\right)^{2} \leq\left(\frac{(M+m)^{2}}{4 M m}\right)^{2}(\Phi(A) \sharp \Phi(B))^{2} .
$$

$\mathrm{Fu}$ and He [5, Theorem 4] generalized (1.2) and (1.3) to the power of $p(p \geq 2)$ :

$$
\Phi\left(\frac{A+B}{2}\right)^{p} \leq\left(\frac{(M+m)^{2}}{4^{\frac{p}{2}} M m}\right)^{p} \Phi(A \sharp B)^{p},
$$

and

$$
\Phi\left(\frac{A+B}{2}\right)^{p} \leq\left(\frac{(M+m)^{2}}{4^{\frac{p}{2}} M m}\right)^{p}(\Phi(A) \sharp \Phi(B))^{p} .
$$

We will improve (1.4) and (1.5) if the condition number $\frac{M}{m}$ is not too big.

Bhatia and Davis [3] proved an operator Wielandt inequality which states that if $0<m \leq A \leq M$ and $X$ and $Y$ are two partial isometries on $\mathcal{H}$ whose final spaces are orthogonal to each other, then for every 2-positive linear map $\Phi$,

$$
\Phi\left(X^{*} A Y\right) \Phi\left(Y^{*} A Y\right)^{-1} \Phi\left(Y^{*} A X\right) \leq\left(\frac{M-m}{M+m}\right)^{2} \Phi\left(X^{*} A X\right) .
$$

Lin [8, Conjecture 3.4] conjectured that a stronger result than (1.6) could be true:

$$
\left\|\Phi\left(X^{*} A Y\right) \Phi\left(Y^{*} A Y\right)^{-1} \Phi\left(Y^{*} A X\right) \Phi\left(X^{*} A X\right)^{-1}\right\| \leq\left(\frac{M-m}{M+m}\right)^{2} .
$$

In an attempt of solving this conjecture, $\mathrm{Fu}$ and $\mathrm{He}$ [5, Theorem 5] proved:

$$
\begin{gathered}
\left\|\Phi\left(X^{*} A Y\right) \Phi\left(Y^{*} A Y\right)^{-1} \Phi\left(Y^{*} A X\right) \Phi\left(X^{*} A X\right)^{-1}\right\| \\
\quad \leq \frac{1}{4}\left(\left(\frac{M-m}{M+m}\right)^{2} M+\frac{1}{m}\right)^{2} .
\end{gathered}
$$

We shall generalize and improve (1.7) under some extra conditions. 


\section{MAIN RESULTS}

Two useful lemmas are given before the main theorems are presented.

Lemma 2.1. Let $A, B \geq 0$. Then the following inequality holds:

$$
\|A B\| \leq \frac{1}{4}\|A+B\|^{2} .
$$

Remark 2.2. Lemma 2.1 is essentially due to Bhatia and Kittaneh in [1, Theorem 1]. They stated the result for the finite dimensional case, however, all technical results used to prove this result for operator norm is also true for the infinite dimensional case. Also, we remark that if $A, B$ are compact operators, then a stronger result can be found in [4].

Lemma 2.3. [2, Theorem 3] Let $A, B \geq 0$. Then for $1 \leq r<\infty$

$$
\left\|A^{r}+B^{r}\right\| \leq\left\|(A+B)^{r}\right\| .
$$

Firstly, we give a refinement of the inequality (1.1) when $p \geq 4$.

Theorem 2.4. Let $0<m \leq A, B \leq M$. Then for every positive unital linear map $\Phi$,

$$
\Phi^{p}\left(A^{-1}\right) \leq \frac{\left(M^{2}+m^{2}\right)^{p}}{16 M^{p} m^{p}} \Phi(A)^{-p}, \quad p \geq 4 .
$$

Proof. The claimed inequality is equivalent to

$$
\left\|\Phi^{\frac{p}{2}}\left(A^{-1}\right) \Phi^{\frac{p}{2}}(A)\right\| \leq \frac{\left(M^{2}+m^{2}\right)^{\frac{p}{2}}}{4 M^{\frac{p}{2}} m^{\frac{p}{2}}} .
$$

Compute

$$
\begin{aligned}
\left\|M^{\frac{p}{2}} m^{\frac{p}{2}} \Phi^{\frac{p}{2}}\left(A^{-1}\right) \Phi^{\frac{p}{2}}(A)\right\| & \leq \frac{1}{4}\left\|M^{\frac{p}{2}} m^{\frac{p}{2}} \Phi^{\frac{p}{2}}\left(A^{-1}\right)+\Phi^{\frac{p}{2}}(A)\right\|^{2} \quad \text { By Lemma 2.1 } \\
& \leq \frac{1}{4}\left\|\left(M^{2} m^{2} \Phi^{2}\left(A^{-1}\right)+\Phi^{2}(A)\right)^{\frac{p}{4}}\right\|^{2} \quad \text { By Lemma 2.3 } \\
& =\frac{1}{4}\left\|M^{2} m^{2} \Phi^{2}\left(A^{-1}\right)+\Phi^{2}(A)\right\|^{p / 2} \\
& \leq \frac{1}{4}\left(M^{2}+m^{2}\right)^{p / 2} . \quad \text { By }[9,(4.7)]
\end{aligned}
$$

So

$$
\left\|\Phi^{\frac{p}{2}}\left(A^{-1}\right) \Phi^{\frac{p}{2}}(A)\right\| \leq \frac{1}{4} \frac{\left(M^{2}+m^{2}\right)^{p / 2}}{M^{\frac{p}{2}} m^{\frac{p}{2}}} .
$$

Thus (2.1) holds.

Remark 2.5. Since $M, m>0$, then

$$
M^{2}+m^{2}<(M+m)^{2} .
$$

Thus, our inequality in Theorem 2.4 is tighter than that in (1.1) when $p \geq 4$.

Next, we present an alternative operator reverse AM-GM inequality to the power of $p(p \geq 4)$. 
Theorem 2.6. Let $0<m \leq A, B \leq M$ and $4 \leq p<\infty$. Then for every positive unital linear map $\Phi$

$$
\Phi^{p}\left(\frac{A+B}{2}\right) \leq \frac{\left(k\left(M^{2}+m^{2}\right)\right)^{p}}{16 M^{p} m^{p}} \Phi^{p}(A \sharp B),
$$

and

$$
\Phi^{p}\left(\frac{A+B}{2}\right) \leq \frac{\left(k\left(M^{2}+m^{2}\right)\right)^{p}}{16 M^{p} m^{p}}(\Phi(A) \sharp \Phi(B))^{p},
$$

where $k=\frac{(M+m)^{2}}{4 M m}$.

Proof. It follows from the inequality (1.2) and operator reverse monotonicity of the inverse that

$$
k^{2} \Phi^{-2}\left(\frac{A+B}{2}\right) \geq \Phi^{-2}(A \sharp B) .
$$

Combining Lemma 2.1, Lemma 2.3 and (2.4) gives

$$
\begin{aligned}
\| \Phi^{\frac{p}{2}}\left(\frac{A+B}{2}\right) M^{\frac{p}{2}} & m^{\frac{p}{2}} \Phi^{-\frac{p}{2}}(A \sharp B) \| \\
& \leq \frac{1}{4}\left\|k^{\frac{p}{4}} \Phi^{\frac{p}{2}}\left(\frac{A+B}{2}\right)+\left(\frac{M^{2} m^{2}}{k}\right)^{\frac{p}{4}} \Phi^{-\frac{p}{2}}(A \sharp B)\right\|^{2} \\
& \leq \frac{1}{4}\left\|\left(k \Phi^{2}\left(\frac{A+B}{2}\right)+\frac{M^{2} m^{2}}{k} \Phi^{-2}(A \sharp B)\right)^{\frac{p}{4}}\right\|^{2} \\
& =\frac{1}{4}\left\|\left(k \Phi^{2}\left(\frac{A+B}{2}\right)+\frac{M^{2} m^{2}}{k} \Phi^{-2}(A \sharp B)\right)\right\|^{\frac{p}{2}} \\
& \leq \frac{1}{4}\left\|\left(k \Phi^{2}\left(\frac{A+B}{2}\right)+k M^{2} m^{2} \Phi^{-2}\left(\frac{A+B}{2}\right)\right)\right\|^{\frac{p}{2}} \\
& \leq \frac{1}{4}\left(k\left(M^{2}+m^{2}\right)\right)^{\frac{p}{2}}, \quad \operatorname{By}[9,(4.7)]
\end{aligned}
$$

which leads to

$$
\left\|\Phi^{\frac{p}{2}}\left(\frac{A+B}{2}\right) \Phi^{-\frac{p}{2}}(A \sharp B)\right\| \leq \frac{1}{4}\left(\frac{k\left(M^{2}+m^{2}\right)}{M m}\right)^{\frac{p}{2}} .
$$

Hence, (2.2) holds.

Similarly, (2.3) holds by the inequality (1.3).

Remark 2.7. When $\frac{M}{m}<2+\sqrt{3}$, we have

$$
\frac{(M+m)^{2}}{4 M m}\left(M^{2}+m^{2}\right)<(M+m)^{2} .
$$

Thus, our inequalities in (2.2) and (2.3) are sharper than those in (1.4) and (1.5), respectively when $\frac{M}{m}<2+\sqrt{3}$ and $p \geq 4$.

Recall that an isometry $X$ on $\mathcal{H}$ is an operator satisfying $X^{*} X=I$. Clearly $X^{*} Y=0$ if and only the ranges of $X, Y$ are orthogonal to each other. Using a similar idea of $\mathrm{Fu}$ and $\mathrm{He}$ [5], we can generalize the inequality (1.7) as follows. 
Theorem 2.8. Let $0<m \leq A \leq M$ and let $X$ and $Y$ be two isometries, whose ranges are orthogonal to each other. Then for every 2-positive unital linear map $\Phi$

$$
\begin{aligned}
& \left\|\left(\Phi\left(X^{*} A Y\right) \Phi\left(Y^{*} A Y\right)^{-1} \Phi\left(Y^{*} A X\right)\right)^{\frac{p}{2}} \Phi\left(X^{*} A X\right)^{-\frac{p}{2}}\right\| \\
& \leq \begin{cases}\left(\frac{M-m}{M+m}\right)^{p}, & \text { when } 0 \leq p \leq 1, \\
\frac{1}{2^{p}}\left(\left(\frac{M-m}{M+m}\right)^{2} M+\frac{1}{m}\right)^{p}, & \text { when } 1<p<2, \\
\frac{1}{4}\left(\left(\frac{M-m}{M+m}\right)^{2} M+\frac{1}{m}\right)^{p}, & \text { when } p \geq 2 .\end{cases}
\end{aligned}
$$

Proof. There are three cases to consider.

Case 1. Combining the inequality (1.6) and the fact that $f(t)=t^{r}$ is operator monotone for $0 \leq r \leq 1$, we have

$$
\left(\Phi\left(X^{*} A Y\right) \Phi\left(Y^{*} A Y\right)^{-1} \Phi\left(Y^{*} A X\right)\right)^{p} \leq\left(\frac{M-m}{M+m}\right)^{2 p} \Phi\left(X^{*} A X\right)^{p},
$$

where $0 \leq p \leq 1$.

From (2.5), we derive

$$
\left\|\left(\Phi\left(X^{*} A Y\right) \Phi\left(Y^{*} A Y\right)^{-1} \Phi\left(Y^{*} A X\right)\right)^{\frac{p}{2}} \Phi\left(X^{*} A X\right)^{-\frac{p}{2}}\right\| \leq\left(\frac{M-m}{M+m}\right)^{p},
$$

when $0 \leq p \leq 1$.

Case 2. Let $\alpha=\left(\frac{M-m}{M+m}\right)^{2} M+\frac{1}{m}$. From the inequality (1.7), we know that

$$
\left(\Phi\left(X^{*} A Y\right) \Phi\left(Y^{*} A Y\right)^{-1} \Phi\left(Y^{*} A X\right)\right)^{2} \leq \frac{1}{16} \alpha^{4} \Phi\left(X^{*} A X\right)^{2},
$$

which together with the fact that $f(t)=t^{r}$ is operator monotone for $0 \leq r \leq 1$ gives

$$
\left(\Phi\left(X^{*} A Y\right) \Phi\left(Y^{*} A Y\right)^{-1} \Phi\left(Y^{*} A X\right)\right)^{p} \leq \frac{1}{4^{p}} \alpha^{2 p} \Phi\left(X^{*} A X\right)^{p},
$$

when $0<p \leq 2$,

i.e.,

$$
\left\|\left(\Phi\left(X^{*} A Y\right) \Phi\left(Y^{*} A Y\right)^{-1} \Phi\left(Y^{*} A X\right)\right)^{\frac{p}{2}} \Phi\left(X^{*} A X\right)^{-\frac{p}{2}}\right\| \leq \frac{1}{2^{p}} \alpha^{p} .
$$

Thus, (2.6) holds when $1<p<2$.

Case 3. When $p \geq 2$, it follows from Lemma 2.1, Lemma 2.3 and the inequality (1.6) that 


$$
\begin{aligned}
\left\|\left(\Phi\left(X^{*} A Y\right) \Phi\left(Y^{*} A Y\right)^{-1} \Phi\left(Y^{*} A X\right)\right)^{\frac{p}{2}} \Phi\left(X^{*} A X\right)^{-\frac{p}{2}}\right\| \\
\leq \frac{1}{4}\left\|\left(\Phi\left(X^{*} A Y\right) \Phi\left(Y^{*} A Y\right)^{-1} \Phi\left(Y^{*} A X\right)\right)^{\frac{p}{2}}+\Phi\left(X^{*} A X\right)^{-\frac{p}{2}}\right\|^{2} \\
\leq \frac{1}{4}\left\|\left(\Phi\left(X^{*} A Y\right) \Phi\left(Y^{*} A Y\right)^{-1} \Phi\left(Y^{*} A X\right)+\Phi\left(X^{*} A X\right)^{-1}\right)^{\frac{p}{2}}\right\|^{2} \\
=\frac{1}{4}\left\|\Phi\left(X^{*} A Y\right) \Phi\left(Y^{*} A Y\right)^{-1} \Phi\left(Y^{*} A X\right)+\Phi\left(X^{*} A X\right)^{-1}\right\|^{p} \\
\leq \frac{1}{4}\left\|\left(\frac{M-m}{M+m}\right)^{2} \Phi\left(X^{*} A X\right)+\Phi\left(X^{*} A X\right)^{-1}\right\|^{p} \\
\leq \frac{1}{4}\left(\left(\frac{M-m}{M+m}\right)^{2} M+\frac{1}{m}\right)^{p} .
\end{aligned}
$$

The last inequality above holds since $X^{*} X=I$ and $0<m \leq A \leq M$, then $m \leq \Phi\left(X^{*} A X\right) \leq M$ and $\frac{1}{M} \leq \Phi\left(X^{*} A X\right)^{-1} \leq \frac{1}{m}$.

It is clear that when $0 \leq p \leq 1$, the bound in Theorem 2.8 is optimal. The next result gives a strengthening of Theorem 2.8 in the case $p>1$.

Theorem 2.9. Let $0<m \leq A \leq M$ and let $X$ and $Y$ be two isometries, whose ranges are orthogonal to each other. Then for $p>1$ and every 2-positive unital linear map $\Phi$

$$
\left\|\left(\Phi\left(X^{*} A Y\right) \Phi\left(Y^{*} A Y\right)^{-1} \Phi\left(Y^{*} A X\right)\right)^{\frac{p}{2}} \Phi\left(X^{*} A X\right)^{-\frac{p}{2}}\right\| \leq\left(\frac{M-m}{M+m}\right)^{p}\left(\frac{M}{m}\right)^{\frac{p}{2}} .
$$

Proof. \| $\left(\Phi\left(X^{*} A Y\right) \Phi\left(Y^{*} A Y\right)^{-1} \Phi\left(Y^{*} A X\right)\right)^{\frac{p}{2}} \Phi\left(X^{*} A X\right)^{-\frac{p}{2}} \|$

$$
\begin{aligned}
& \leq\left\|\left(\Phi\left(X^{*} A Y\right) \Phi\left(Y^{*} A Y\right)^{-1} \Phi\left(Y^{*} A X\right)\right)^{\frac{p}{2}}\right\|\left\|\Phi\left(X^{*} A X\right)^{-\frac{p}{2}}\right\| \\
& =\left\|\Phi\left(X^{*} A Y\right) \Phi\left(Y^{*} A Y\right)^{-1} \Phi\left(Y^{*} A X\right)\right\|^{\frac{p}{2}}\left\|\Phi\left(X^{*} A X\right)^{-1}\right\|^{\frac{p}{2}} \\
& \leq\left(\frac{M-m}{M+m}\right)^{p}\left\|\Phi\left(X^{*} A X\right)\right\|^{\frac{p}{2}}\left\|\Phi\left(X^{*} A X\right)^{-1}\right\|^{\frac{p}{2}} \\
& \leq\left(\frac{M-m}{M+m}\right)^{p}\left(\frac{M}{m}\right)^{\frac{p}{2}} .
\end{aligned}
$$

The required result follows.

Remark 2.10. By the scalar AM-GM inequality, it is easy to see that the bound in Theorem 2.9 is tighter than the corresponding bounds in Theorem 2.8.

\section{ACKNOWLEDGEMENTS}

The author thanks the referee and the handling editor for their valuable comments and suggestions which improve the presentation of the paper. This work was supported in part by the Foundation of Chongqing University of Posts and Telecommunications for the Scholars with Doctorate (Grant No. A2014016) and in part by the Basic and Advanced Research Project of CQ CSTC (Grant No. cstc2014jcyjA00044). 


\section{REFERENCES}

1. R. Bhatia and F. Kittaneh, Notes on matrix arithmetic-geometric mean inequalities, Linear Algebra Appl. 308 (2000), 203-211.

2. R. Bhatia and F. Kittaneh, Norm inequalities for positive operators, Lett. Math. Phys. 43 (1998), 225-231.

3. R. Bhatia and C. Davis, More operator versions of the Schwarz inequality, Comm. Math. Phys. 215 (2000), 239-244.

4. S.W. Drury, On a question of Bhatia and Kittaneh, Linear Algebra Appl. 437 (2012) 19551960.

5. X. Fu and C. He, Some operator inequalities for positive linear maps, Linear Multilinear Algebra (2014), doi:10.1080/03081087.2014.880432.

6. R.A. Horn and C.R. Johnson, Matrix Analysis, Cambridge University Press, 1985.

7. L.V. Kantorovich, Functional analysis and applied mathematics (in Russian), Uspehi Matem. Nauk(N.S.) 3 (1948), 89-185.

8. M. Lin, On an operator Kantorovich inequality for positive linear maps, J. Math. Anal. Appl. 402 (2013), 127-132.

9. M. Lin, Squaring a revese AM-GM inequality, Studia Math., 215 (2013), 187-194.

10. A.W. Marshall and I. Olkin, Matrix versions of the Cauchy and Kantorovich inequalities, Aequationes Math. 40 (1990), 89-93.

11. M.S. Moslehian, Recent developments of the operator Kantorovich inequality, Expo. Math. 30 (2012), 376-388.

12. M.S. Moslehian and J.I. Fujii, Operator inequalities related to weak 2-positivity, J. Math. Inequal. 7 (2013), 175-182.

Faculty of science, Chongqing University of Posts and Telecommunications, Chongqing 400065, China.

E-mail address: zhpp040102480126.com 